\title{
THE FIXED-POINT PROPERTY IN BANACH SPACES CONTAINING A COPY OF $c_{0}$
}

\author{
MARIA A. JAPÓN PINEDA
}

Received 6 July 2001

We prove that every Banach space containing an isomorphic copy of $c_{0}$ fails to have the fixed-point property for asymptotically nonexpansive mappings with respect to some locally convex topology which is coarser than the weak topology. If the copy of $c_{0}$ is asymptotically isometric, this result can be improved, because we can prove the failure of the fixed-point property for nonexpansive mappings.

\section{Introduction}

Let $X$ be a Banach space and $\Sigma$ a class of mappings defined from closed convex bounded subsets of $X$ into itself. We say that $X$ has the fixed-point property (FPP) for the class $\Sigma$ if every mapping, belonging to $\Sigma$, has a fixed point. If the Banach space is endowed with a topology $\tau$, we say that $X$ has the $\tau$-FPP for the class $\Sigma$ if every mapping in $\Sigma$ defined on a $\tau$-compact convex set has a fixed point.

In this paper, we consider the class of asymptotically nonexpansive mappings and the class of nonexpansive mappings. Recall that a mapping $T: C \rightarrow C$ is said to be asymptotically nonexpansive, if there exists a real sequence $\left\{k_{n}\right\}$ such that $\lim _{n} k_{n}=1$, and $\left\|T^{n} x-T^{n} y\right\| \leq k_{n}\|x-y\|$ for every $x, y \in C$. In particular, if $\|T x-T y\| \leq\|x-y\|$ for all $x, y \in C, T$ is said to be nonexpansive.

It is well known that the existence of fixed points for nonexpansive, or asymptotically nonexpansive mappings, strongly depends on the geometrical properties of the Banach space $X$ (see, e.g., $[1,3,10,11,16,17,21,22]$, and the references therein). On the other hand, it is not difficult to check that the Banach spaces $c_{0}$ and $\ell_{1}$ fail to have the FPP for nonexpansive mappings. However, it is an open problem, if this failure can be extended to Banach spaces containing an isomorphic copy of either $\ell_{1}$ or $c_{0}$. A partial answer to this problem was given in $[7,8]$, where the authors proved that if a Banach space contains an asymptotically isometric copy of either $\ell_{1}$ or $c_{0}$, then it fails to have the FPP for 
nonexpansive mappings. With respect to the class of asymptotically nonexpansive mappings, it has been recently proved [9] that every Banach space containing an isomorphic copy of $c_{0}$ fails to have the FPP for this class of mappings. It is unknown if the same holds for Banach spaces which contain an isomorphic copy of $\ell_{1}$.

It was proved by Maurey [19] that, when $c_{0}$ is endowed with its weak topology, $c_{0}$ has the $w$-FPP for nonexpansive mappings. However, it seems to be an open problem, if the same property holds for asymptotically nonexpansive mappings. From Maurey's result, we can deduce that $c_{0}$ has the $\tau$-FPP for nonexpansive mappings whenever $\tau$ is any topology finer than the weak topology. Thus, the following question could be raised: if we consider $c_{0}$ endowed with a locally convex topology $\tau$ coarser than the weak topology, does $c_{0}$ have the $\tau$-FPP for nonexpansive mappings? The answer to this question was given in a negative way by Llorens-Fuster and Sims [18] who proved that $c_{0}$ can be endowed with a locally convex topology $\tau$, which is slightly coarser than the weak topology, and for which $c_{0}$ fails to have the $\tau$-FPP for nonexpansive mappings. This shows the instability of the FPP with respect to the considered topology.

In this paper, we consider Banach spaces which contain a copy of $c_{0}$, and we extend the construction given in [18] to this class of spaces. Firstly, we prove that every Banach space containing an isomorphic copy of $c_{0}$ fails to have the FPP for asymptotically nonexpansive mappings with respect to some locally convex topology coarser than the weak topology. Secondly, if the Banach space contains an asymptotically isometric copy of $c_{0}$, we check that this result can be improved, because we can prove the failure of the fixed-point property for nonexpansive mappings. Notice that there exist some examples of Banach spaces containing asymptotically isometric copies of $c_{0}$ with different behaviour with respect to the $w$-FPP for nonexpansive mappings. However, we prove that they always fail to have the FPP for nonexpansive mappings with respect to some locally convex topology which is slightly coarser than the weak topology. This fact answers in a negative way to the question of whether the FPP for nonexpansive mappings can be extended to locally convex topologies coarser than the weak topology for this class of Banach spaces.

\section{Preliminaries}

James [14] proved that if a Banach space $X$ contains a subspace which is isomorphic to $c_{0}$, then it contains almost isometric copies of $c_{0}$, that is, for every $\epsilon>0$ there exists a sequence $\left\{x_{n}\right\} \subset X$, so that

$$
(1-\epsilon) \sup _{n}\left|t_{n}\right| \leq\left\|\sum_{n=1}^{\infty} t_{n} x_{n}\right\| \leq \sup _{n}\left|t_{n}\right|
$$

for every $\left\{t_{n}\right\} \in c_{0}$. 
It is very well known that the Banach space $c_{0}$ fails to have the FPP for nonexpansive mappings [15], which implies that every Banach space containing an isometric copy of $c_{0}$ also fails to have this property. James' result [14] induces to think that Banach spaces containing a copy of $c_{0}$ could also fail to have the FPP for nonexpansive mappings. However, this problem is still open. In [8] the authors give a partial answer showing that when the Banach space $X$ contains a copy of $c_{0}$, which is a good copy, $X$ does fail to have the FPP for nonexpansive mappings. These copies of $c_{0}$ are good in the following sense.

Definition 2.1. A Banach space $X$ is said to contain an asymptotically isometric copy of $c_{0}$ if there exists a null sequence $\left\{\epsilon_{n}\right\} \in(0,1)$ and a sequence $\left\{x_{n}\right\} \subset X$, so that

$$
\sup _{n}\left(1-\epsilon_{n}\right)\left|t_{n}\right| \leq\left\|\sum_{n=1}^{\infty} t_{n} x_{n}\right\| \leq \sup _{n}\left|t_{n}\right|
$$

for every $\left\{t_{n}\right\} \in c_{0}$.

It is clear that a Banach space containing an asymptotically isometric copy of $c_{0}$, also contains an isomorphic copy of $c_{0}$. However, the converse is not true; there exist renormings of $c_{0}$ which contain no asymptotically isometric copy of $c_{0}[6]$.

On the other hand, with respect to asymptotically nonexpansive mappings, it has been proved [9] that a Banach space containing an isomorphic copy of $c_{0}$ fails to have the FPP for this class of mappings. In order to do that, the authors improve James' result and obtained the following theorem.

Theorem 2.2. A Banach space contains an isomorphic copy of $c_{0}$ if and only if there exist a null sequence $\left\{\epsilon_{n}\right\}$ in $(0,1)$ and a sequence $\left\{x_{n}\right\}$ in $X$, so that

$$
\sup _{n \geq k}\left|t_{n}\right| \leq\left\|\sum_{n=k}^{\infty} t_{n} x_{n}\right\| \leq\left(1+\epsilon_{k}\right) \sup _{n \geq k}\left|t_{n}\right|
$$

for all $\left\{t_{n}\right\}_{n} \in c_{0}$ and for all $k \in \mathbb{N}$.

\section{Banach spaces containing an isomorphic copy of $c_{0}$}

In this section, we consider a Banach space $X$ containing an isomorphic copy of $c_{0}$, and we prove that there exists a locally convex topology $\tau$ on $X$, coarser than the weak topology, and such that $X$ fails to have the $\tau$-FPP for asymptotically nonexpansive mappings.

We begin by defining the locally convex topology, for which we are going to prove the main result in this section.

Let $\left\{e_{n}\right\}$ denote the canonical basis of $c_{0}$ and $\left\{d_{n}\right\}$ the summing basis of $c_{0}$, that is, $d_{n}:=e_{1}+e_{2}+\cdots+e_{n}$ for every $n \in \mathbb{N}$. 
Let $\left\{x_{n}\right\}$ be the sequence in $X$ satisfying Theorem 2.2 and define

$$
w_{n}:=x_{1}+x_{2}+\cdots+x_{n}
$$

for every $n \in \mathbb{N}$. Thus, $\left\{w_{n}\right\}$ is a basic sequence equivalent to the summing basis of $c_{0}$.

Let $Y$ be the closed subspace of $X$ spanned by the sequence $\left\{x_{n}\right\}$, and let $H$ be the isomorphism from $c_{0}$ into $X$ (and onto $Y$ ) which maps $e_{n}$ to $x_{n}$ for every $n \in$ $\mathbb{N}$. Since the sequence $\left\{d_{n}\right\}$ is $\sigma\left(\ell_{\infty}, \ell_{1}\right)$ convergent to the vector $\hat{d}=(1,1,1, \ldots) \in$ $\ell_{\infty} \backslash c_{0}$, the sequence $\left\{w_{n}\right\}$ is $\sigma\left(X^{\star \star}, X^{\star}\right)$ convergent to $\hat{w}=H^{\star \star}(\hat{d}) \in X^{\star \star}$, since $H^{\star \star}$ is $w^{\star}$-to $w^{\star}$-continuous. Moreover, since $H^{\star \star}\left(\ell_{\infty}\right) \subset Y^{\perp \perp}$, it follows that $H^{\star \star}\left(\ell_{\infty}\right) \cap X=Y=H\left(c_{0}\right)$. Thus $\hat{w} \notin X$.

Using [12, Lemma 1.1.11], we deduce that the subspace

$$
\mathscr{E}:=\operatorname{ker}\{\hat{w}\}=\left\{x^{\star} \in X^{\star}: \hat{w}\left(x^{\star}\right)=0\right\}
$$

is a norming space for $X$ and consequently $\mathscr{E}$ separates points of $X$. Thus, we can endow $X$ with the topology $\sigma(X, \mathscr{E})$, which is the weakest locally convex linear topology on $X$ making continuous all the elements of $\mathscr{E}$. This topology is slightly coarser than the weak topology since it is induced by a norming codimensionone subspace of $X^{\star}$.

The main result of this section is the following theorem.

Theorem 3.1. Let $X$ be a Banach space containing a copy of $c_{0}$. Endow $X$ with the topology $\sigma(X, \mathscr{E})$. Then $X$ fails to have the $\sigma(X, \mathscr{E})$-FPP for asymptotically nonexpansive mappings.

Proof. Define the set

$$
C:=\left\{\sum_{n=1}^{\infty} t_{n} w_{n}: 0 \leq t_{n} \leq 1, \sum_{n=1}^{\infty} t_{n} \leq 1\right\},
$$

and the mapping $T: C \rightarrow C$ by

$$
T\left(\sum_{n=1}^{\infty} t_{n} w_{n}\right)=\left(1-\sum_{n=1}^{\infty} t_{n}\right) w_{1}+\sum_{n=1}^{\infty} t_{n} w_{n+1} .
$$

We are going to prove that $C$ is a convex bounded $\sigma(X, \mathscr{E})$-compact subset of $X$, and that $T$ is a fixed-point free asymptotically nonexpansive mapping.

(a) The set $C$ is a convex bounded $\sigma(X, \mathscr{E})$-compact subset of $X$.

It is clear that $C$ is a convex bounded subset of $X$. We prove that $C$ is $\sigma(X, \mathscr{E})$ compact. Fix $\left\{y_{\alpha}\right\}_{\alpha}$ a net in $C$. We can write

$$
y_{\alpha}=\sum_{n=1}^{\infty} t_{n}^{(\alpha)} w_{n}
$$

where $0 \leq t_{n}^{(\alpha)} \leq 1$ and $\sum_{n=1}^{\infty} t_{n}^{(\alpha)} \leq 1$ for all $\alpha$. 
Define $t^{(\alpha)}=\left\{t_{n}^{(\alpha)}\right\}_{n \geq 1} \in \ell_{1}$. In fact, $\left\{t^{(\alpha)}\right\}_{\alpha}$ is a net in the closed unit ball of $\ell_{1}$, which is $\sigma\left(\ell_{1}, c_{0}\right)$ compact by Banach-Alaoglu theorem. Thus, without loss of generality, we can assume that $\left\{t^{(\alpha)}\right\}_{\alpha}$ itself converges with respect to the $\sigma\left(\ell_{1}, c_{0}\right)$ topology (which coincides with the topology of coordinatewise convergence on the unit ball of $\ell_{1}$ ) to some $\lambda=\left\{\lambda_{n}\right\}_{n \geq 1} \in \ell_{1}$. Moreover, it follows that $\lambda_{n} \geq 0$ for all $n \geq 1$, and by the $\sigma\left(\ell_{1}, c_{0}\right)$-lower semicontinuity of the norm, we deduce

$$
\sum_{n=1}^{\infty} \lambda_{n} \leq \liminf _{\alpha} \sum_{n=1}^{\infty} t_{n}^{(\alpha)} \leq 1 .
$$

Define

$$
y:=\sum_{n=1}^{\infty} \lambda_{n} w_{n}
$$

which belongs to $C$. We prove that $\left\{y_{\alpha}\right\}_{\alpha}$ is $\sigma(X, \mathscr{E})$-convergent to $y$.

Fix $x^{\star} \in \mathscr{E}$, so that $\hat{w}\left(x^{\star}\right)=0$. Recall that $H$ maps $c_{0}$ into $X, Y=H\left(c_{0}\right)$, and $H\left(e_{n}\right)=x_{n}$, for all $n \geq 1$. Let $\left\{y_{m}^{\star}\right\}_{m \geq 1}$ be the basic sequence of linear functionals in $Y^{\star}$ dual to $\left\{x_{n}\right\}_{n \geq 1}$; that is, $y_{m}^{\star}\left(x_{n}\right)=\delta_{m, n}$. Note that each $\left\|y_{m}^{\star}\right\| \leq 1$. For each $m$, let $x_{m}^{\star}$ be a Hahn-Banach extension of $y_{m}^{\star}$ to $X^{\star}$, and note that $H^{\star}$ maps $X^{\star}$ onto $\ell_{1}$. Now, we define $s_{n}:=x^{\star}\left(x_{n}\right)$ for all $n \geq 1$. It is easy to directly check that $s:=\left\{s_{n}\right\}_{n \geq 1}$ is an element of $\ell_{1}$. Thus we may define $y^{\star}:=$ $\sum_{m \geq 1} s_{m} x_{m}^{\star} \in X^{\star}$; while a simple calculation shows that $H^{\star}\left(y^{\star}\right)=s$. Next, define $y^{\perp}:=x^{\star}-y^{\star} \in X^{\star}$. By the definition of $s=\left\{s_{n}\right\}_{n \geq 1}$, we have $y^{\perp} \in Y^{\perp}$. But $\hat{w} \in H^{\star \star}\left(\ell_{\infty}\right) \subset Y^{\perp \perp}$, and so $\hat{w}\left(y^{\perp}\right)=0$, consequently, $\hat{w}\left(y^{\star}\right)=0$. Finally, $0=\hat{w}\left(y^{\star}\right)=\left(H^{\star \star}(\hat{d})\right)\left(y^{\star}\right)=\hat{d}\left(H^{\star}\left(y^{\star}\right)\right)=\hat{d}(s)=\sum_{n \geq 1} s_{n}$. Thus, the sequence $\left\{\sum_{i=1}^{n} s_{i}\right\}_{n \geq 1}$ belongs to $c_{0}$.

On the other hand, notice the following

$$
\begin{gathered}
x^{\star}\left(y_{\alpha}\right)=y^{\star}\left(y_{\alpha}\right)=H^{\star} y^{\star}\left(H^{-1} y_{\alpha}\right)=\sum_{n=1}^{\infty} t_{n}^{(\alpha)}\left[\sum_{i=1}^{n} s_{i}\right], \\
x^{\star}(y)=y^{\star}(y)=H^{\star} y^{\star}\left(H^{-1} y\right)=\sum_{n=1}^{\infty} \lambda_{n}\left[\sum_{i=1}^{n} s_{i}\right] .
\end{gathered}
$$

Since $\left\{t^{(\alpha)}\right\}_{\alpha}$ tends to $\lambda=\left\{\lambda_{n}\right\}_{n}$ in the $\sigma\left(\ell_{1}, c_{0}\right)$ topology, we have that $x^{\star}\left(y_{\alpha}\right)$ tends to $x^{\star}(y)$ and $C$ is $\sigma(X, \mathscr{E})$-compact.

(b) The mapping $T$ is a fixed-point free asymptotically nonexpansive mapping.

It is clear that $T$ maps $C$ into $C$ and $T$ is fixed-point free. Indeed, if $x=$ $\sum_{n=1}^{\infty} t_{n} w_{n} \in C$ satisfies $T x=x$, then $t_{n}=t_{m}$ for every $n, m \in \mathbb{N}$, which implies that $t_{n}=0$ for every $n \in \mathbb{N}$. But the equality $T x=x$ also implies that $\sum_{n=1}^{\infty} t_{n}=1$ which is a contradiction.

We prove that $T$ is an asymptotically nonexpansive mapping. 
Fix $k \in \mathbb{N}$ and $x=\sum_{n=1}^{\infty} t_{n} w_{n} \in C$. It is not difficult to check that

$$
T^{k}(x)=\left(1-\sum_{n=1}^{\infty} t_{n}\right) w_{k}+\sum_{n=1}^{\infty} t_{n} w_{n+k}=x_{1}+\cdots+x_{k}+\sum_{n=1}^{\infty}\left[\sum_{i=n}^{\infty} t_{i}\right] x_{n+k} .
$$

Now let $x, y$ be two elements in $C, x=\sum_{n=1}^{\infty} t_{n} w_{n}, y=\sum_{n=1}^{\infty} s_{n} w_{n}$

$$
\begin{aligned}
\left\|T^{k} x-T^{k} y\right\| & =\left\|\sum_{n=1}^{\infty}\left[\sum_{i=n}^{\infty}\left(t_{i}-s_{i}\right)\right] x_{n+k}\right\| \\
& \leq\left(1+\epsilon_{k+1}\right) \sup _{n \geq 1}\left|\sum_{i=n}^{\infty}\left(t_{i}-s_{i}\right)\right| \\
& \leq\left(1+\epsilon_{k+1}\right)\left\|\sum_{n=1}^{\infty}\left[\sum_{i=n}^{\infty}\left(t_{i}-s_{i}\right)\right] x_{n}\right\| \\
& =\left(1+\epsilon_{k+1}\right)\left\|\sum_{n=1}^{\infty}\left(t_{n}-s_{n}\right) w_{n}\right\| \\
& =\left(1+\epsilon_{k+1}\right)\|x-y\| .
\end{aligned}
$$

Since $1+\epsilon_{k+1} \rightarrow 1$ as $k \rightarrow \infty$, this shows that $T$ is an asymptotically nonexpansive mapping, and the proof of the theorem is complete.

Remark 3.2. It is an open question if the assertion of Theorem 3.1 holds when the weak topology is considered. In fact, it is unknown if $c_{0}$ itself, or some Banach space containing a copy of $c_{0}$, satisfies the $w$-FPP for asymptotically nonexpansive mappings.

Remark 3.3. Notice that the subset $C$ is also $\sigma(X, \mathscr{E})$-sequentially compact. This can be shown using the same arguments since the unit ball of $\ell_{1}$ is $\sigma\left(\ell_{1}, c_{0}\right)$ sequentially compact. Therefore, we see that $X$ also fails to have the fixed-point property for asymptotically nonexpansive mappings with respect to $\sigma(X, \mathscr{E})$ for domains which are convex bounded and $\sigma(X, \mathscr{E})$-sequentially compact (see [4]).

\section{Banach spaces containing an asymptotically isometric copy of $c_{0}$}

In this section, we consider Banach spaces containing an asymptotically isometric copy of $c_{0}$. In this case, we prove that Theorem 3.1 can be improved in the setting of nonexpansive mappings.

We begin by defining the locally convex topology, for which we later prove that $X$ fails to have the FPP for nonexpansive mappings. 
Let $X$ be a Banach space containing an asymptotically isometric copy of $c_{0}$, and let $\left\{x_{n}\right\}$ be the sequence in $X$ such that

$$
\sup _{n}\left(1-\epsilon_{n}\right)\left|t_{n}\right| \leq\left\|\sum_{n=1}^{\infty} t_{n} x_{n}\right\| \leq \sup _{n}\left|t_{n}\right|
$$

for all $\left\{t_{n}\right\} \in \mathcal{c}_{0}$, being $\left\{\epsilon_{n}\right\}$ a null sequence in $(0,1)$ and each $\epsilon_{n}<1 / 2$. Taking a subsequence we can assume that $\sum_{n=1}^{\infty} \epsilon_{n}<+\infty$, and so $\prod_{n=1}^{\infty}\left(1-2 \epsilon_{n}\right) \in(0,1)$. Defining $\lambda_{n+1}=\left(1-2 \epsilon_{n}\right) \lambda_{n}$ with $\lambda_{1}>1 / \prod_{n=1}^{\infty}\left(1-2 \epsilon_{n}\right)$ we have a sequence $\left\{\lambda_{n}\right\} \subset(1,+\infty)$ such that $\lambda_{n+1}<\left(1-\epsilon_{n}\right) \lambda_{n}$ for every $n \in \mathbb{N}$.

Define $y_{n}:=\lambda_{n} x_{n}$ and let $\left\{w_{n}\right\}$ be the sequence given by

$$
w_{n}:=y_{1}+y_{2}+\cdots+y_{n}
$$

for all $n \in \mathbb{N}$. Notice that $\left\{w_{n}\right\}$ is also a basic sequence.

Let $H: c_{0} \rightarrow X$ be the isomorphism between $c_{0}$ and the closed subspace $Y$ spanned by $\left\{x_{n}\right\}$. Using the same arguments as in Section 3, we can check that $\left\{w_{n}\right\}$ is $\sigma\left(X^{\star \star}, X^{\star}\right)$-convergent to $\hat{w}=T^{\star \star}(\hat{d}) \in X^{\star \star} \backslash X$ where $\hat{d}=\left\{\lambda_{n}\right\}_{n} \in$ $\ell_{\infty}$. Now we can deduce that the subspace

$$
\mathscr{E}=\operatorname{ker}(\hat{w})=\left\{x^{\star} \in X^{\star}: \hat{w}\left(x^{\star}\right)=0\right\}
$$

is a norming space of $X^{\star}$, and the topology $\sigma(X, \mathscr{E})$ is a locally convex topology on $X$, which is coarser than the weak topology.

With respect to $\sigma(X, \mathscr{E})$ we have the main result of this section.

THEOREM 4.1. Let $X$ be a Banach space containing an asymptotically isometric copy of $c_{0}$. Endow $X$ with the $\sigma(X, \mathscr{E})$ topology defined above. Then $X$ fails to have the $\sigma(X, \mathscr{E})-F P P$ for nonexpansive mappings.

Proof. As in the proof of Theorem 3.1 we define

$$
C:=\left\{\sum_{n=1}^{\infty} t_{n} w_{n}: 0 \leq t_{n} \leq 1, \sum_{n=1}^{\infty} t_{n} \leq 1\right\},
$$

and $T: C \rightarrow C$ given by

$$
T\left(\sum_{n=1}^{\infty} t_{n} w_{n}\right)=\left(1-\sum_{n=1}^{\infty} t_{n}\right) w_{1}+\sum_{n=1}^{\infty} t_{n} w_{n+1} .
$$

Using the same arguments as in the proof of Theorem 3.1, it can be proved that $C$ is a convex bounded $\sigma(X, \mathscr{E})$-compact set of $X$, and that $T$ is a fixedpoint free mapping. We prove that $T$ is nonexpansive (in fact $T$ is going to be contractive). 
Fix $x, y \in C$ with $x \neq y, x=\sum_{n=1}^{\infty} t_{n} w_{n}, y=\sum_{n=1}^{\infty} s_{n} w_{n}$. Then we have

$$
\begin{aligned}
\|T x-T y\| & =\left\|\sum_{n=1}^{\infty}\left[\sum_{i=n}^{\infty}\left(s_{i}-t_{i}\right)\right] y_{n+1}\right\| \\
& =\left\|\sum_{n=1}^{\infty}\left[\sum_{i=n}^{\infty}\left(s_{i}-t_{i}\right)\right] \lambda_{n+1} x_{n+1}\right\| \leq \sup _{n \geq 1}\left|\sum_{i=n}^{\infty}\left(s_{i}-t_{i}\right) \lambda_{n+1}\right| \\
& <\sup _{n \geq 1}\left(1-\epsilon_{n}\right)\left|\sum_{i=n}^{\infty}\left(s_{i}-t_{i}\right) \lambda_{n}\right| \leq\left\|\sum_{n=1}^{\infty}\left[\sum_{i=n}^{\infty}\left(s_{i}-t_{i}\right)\right] \lambda_{n} x_{n}\right\| \\
& =\left\|\sum_{n=1}^{\infty}\left[\sum_{i=n}^{\infty}\left(s_{i}-t_{i}\right)\right] y_{n}\right\|=\|x-y\| .
\end{aligned}
$$

This completes the proof.

Remark 4.2. Notice that Theorem 4.1 does not hold for the weak topology. In fact, we can find examples of Banach spaces containing an isometric copy of $c_{0}$, and having different behaviour with respect to the $w$-FPP for nonexpansive mappings. Indeed, the Banach space $c_{0}$ itself has the $w$-FPP for nonexpansive mappings [19]. However, consider now the Banach space $X=c_{0} \oplus_{1} L_{1}([0,1])$. This space has an isometric copy of $c_{0}$ and also an isometric copy of $L_{1}([0,1])$. Using Alspach's result [2] we deduce that $X$ fails to have the $w$-FPP for nonexpansive mappings.

Remark 4.3. Theorem 4.1 extends the construction given in [18] for $c_{0}$ to more general Banach spaces. For instance, $c_{0}$ is a particular example of a Banach space which is $M$-embedded. In general, $M$-embedded Banach spaces (also called $M$ ideals in their bidual) are those Banach spaces $X$ for which the decomposition $X^{\star \star \star}=X^{\star} \oplus_{1} X^{\perp}$ holds (see [13, Chapter III] for more examples and a wide study of this class of Banach spaces). It is proved that every nonreflexive $M$ embedded Banach space contains an asymptotically isometric copy of $c_{0}$ [20]. Therefore, by Theorem 4.1, every $M$-embedded Banach space fails to have the FPP for nonexpansive mappings, with respect to some linear topology coarser than the weak topology. In general, it is unknown if $M$-embedded Banach spaces have the $w$-FPP for nonexpansive mappings.

Remark 4.4. It is an open problem if convex weakly compact subsets of $c_{0}$ can be characterized as the unique convex closed bounded subsets of $c_{0}$, which have the generic fixed-point property for nonexpansive mappings (see [18]). In [5] the authors prove that this characterization is possible if we use the class of affine uniformly Lipschitzian mappings. By [18, Section 3] which Theorem 4.1 generalizes, we see that the question raised in [18] and the results given in [5] cannot be extended to arbitrary locally convex topology coarser than the weak topology, because the mapping $T$ given in $[18$, Section 3$]$ is also affine. 


\section{Acknowledgments}

This research is partially supported by DGICYT PB 96-1338-C02-C01 and J.A. FQM 0127.

\section{References}

[1] A. G. Aksoy and M. A. Khamsi, Nonstandard Methods in Fixed Point Theory, Universitext, Springer-Verlag, New York, 1990.

[2] D. E. Alspach, A fixed point free nonexpansive map, Proc. Amer. Math. Soc. 82 (1981), no. 3, 423-424.

[3] J. M. Ayerbe, T. Domínguez Benavides, and G. López Acedo, Measures of Noncompactness in Metric Fixed Point Theory, Operator Theory: Advances and Applications, vol. 99, Birkhäuser Verlag, Basel, 1997.

[4] T. Domínguez Benavides, J. García Falset, and M. A. Japón Pineda, The $\tau$-fixed point property for nonexpansive mappings, Abstr. Appl. Anal. 3 (1998), no. 3-4, 343362.

[5] T. Domínguez Benavides, M. A. Japón Pineda, and S. Prus, Weak compactness and fixed point property for affine mappings, to appear in J. Funct. Anal.

[6] P. N. Dowling, W. B. Johnson, C. J. Lennard, and B. Turett, The optimality of James's distortion theorems, Proc. Amer. Math. Soc. 125 (1997), no. 1, 167-174.

[7] P. N. Dowling and C. J. Lennard, Every nonreflexive subspace of $L_{1}[0,1]$ fails the fixed point property, Proc. Amer. Math. Soc. 125 (1997), no. 2, 443-446.

[8] P. N. Dowling, C. J. Lennard, and B. Turett, Reflexivity and the fixed-point property for nonexpansive maps, J. Math. Anal. Appl. 200 (1996), no. 3, 653-662.

[9] Some fixed point results in $l^{1}$ and $c_{0}$, Nonlinear Anal. 39 (2000), no. 7, Theory Methods, Ser. A, 929-936.

[10] K. Goebel and W. A. Kirk, A fixed point theorem for asymptotically nonexpansive mappings, Proc. Amer. Math. Soc. 35 (1972), 171-174.

[11] , Topics in Metric Fixed Point Theory, Cambridge Studies in Advanced Mathematics, vol. 28, Cambridge University Press, Cambridge, 1990.

[12] S. Guerre-Delabrière, Classical Sequences in Banach Spaces, Monographs and Textbooks in Pure and Applied Mathematics, vol. 166, Marcel Dekker, New York, 1992.

[13] P. Harmand, D. Werner, and W. Werner, M-Ideals in Banach Spaces and Banach Algebras, Lecture Notes in Mathematics, vol. 1547, Springer-Verlag, Berlin, 1993.

[14] R. C. James, Uniformly non-square Banach spaces, Ann. of Math. (2) 80 (1964), 542 550.

[15] S. Kakutani, Topological properties of the unit sphere of a Hilbert space, Proc. Imp. Acad. Tokyo 19 (1943), 269-271.

[16] T.-H. Kim and H. K. Xu, Remarks on asymptotically nonexpansive mappings, Nonlinear Anal. 41 (2000), no. 3-4, Theory Methods, Ser. A, 405-415.

[17] P.-K. Lin, K.-K. Tan, and H. K. Xu, Demiclosedness principle and asymptotic behavior for asymptotically nonexpansive mappings, Nonlinear Anal. 24 (1995), no. 6, 929946.

[18] E. Llorens-Fuster and B. Sims, The fixed point property in $c_{0}$, Canad. Math. Bull. 41 (1998), no. 4, 413-422. 


\section{The fixed-point property}

[19] B. Maurey, Points fixes des contractions de certains faiblement compacts de $L^{1}$ [Fixed points of the contractions of certain weakly compact subsets of $\left.L^{1}\right]$, Seminar on Functional Analysis, 1980-1981, École Polytechnique, Palaiseau, 1981 (French).

[20] H. Pfitzner, A note on asymptotically isometric copies of $l^{1}$ and $c_{0}$, Proc. Amer. Math. Soc. 129 (2001), no. 5, 1367-1373.

[21] H. K. Xu, Existence and convergence for fixed points of mappings of asymptotically nonexpansive type, Nonlinear Anal. 16 (1991), no. 12, 1139-1146.

[22] Existence and iterative convergence for fixed points of nonlinear mappings, Ph.D. thesis, Xi' an Jiaotong University, China, 1998.

Maria A. Japón Pineda: Departamento de Análisis Matemático, Universidad de Sevilla, 1080 Sevilla, Spain

E-mail address: japon@us.es 


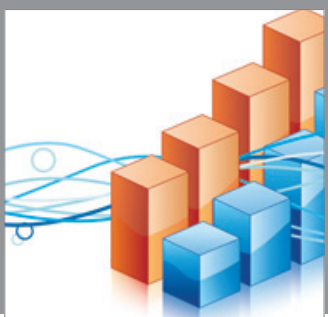

Advances in

Operations Research

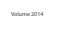

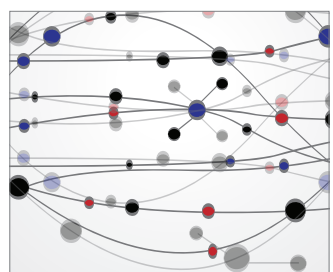

\section{The Scientific} World Journal
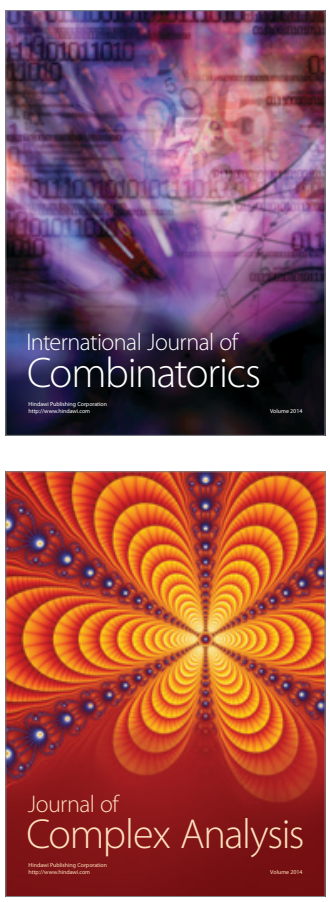

International Journal of

Mathematics and

Mathematical

Sciences
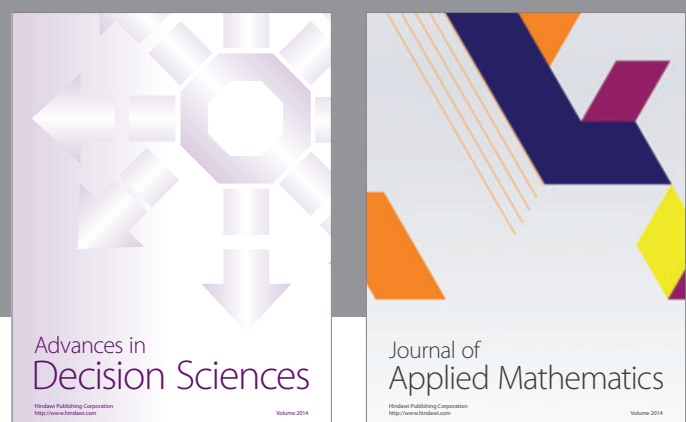

Journal of

Applied Mathematics
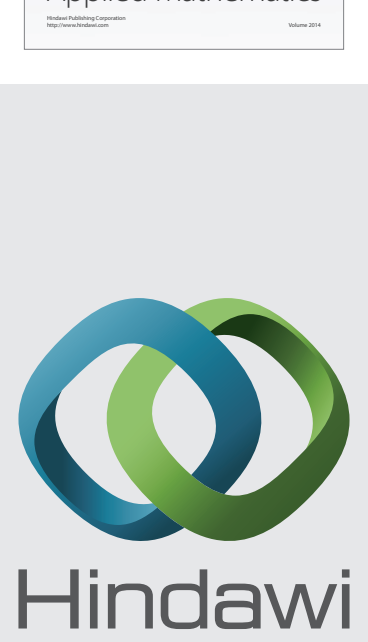

Submit your manuscripts at http://www.hindawi.com
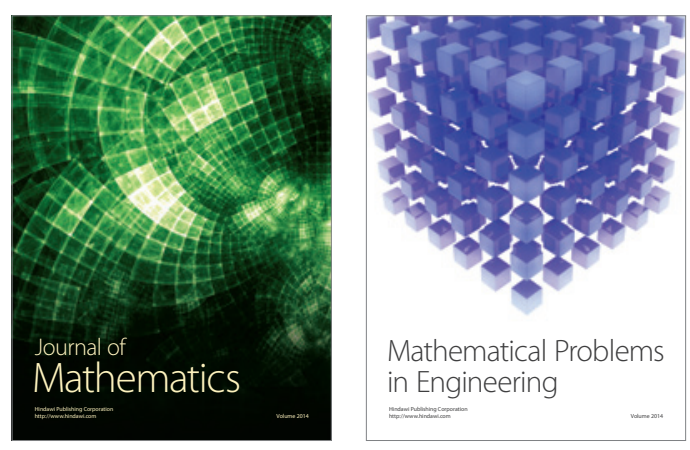

Mathematical Problems in Engineering
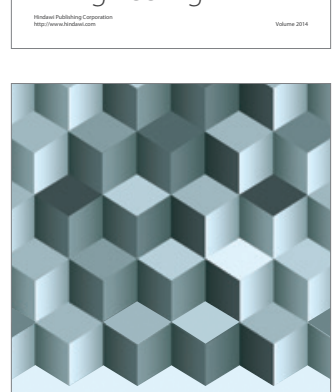

Journal of

Function Spaces
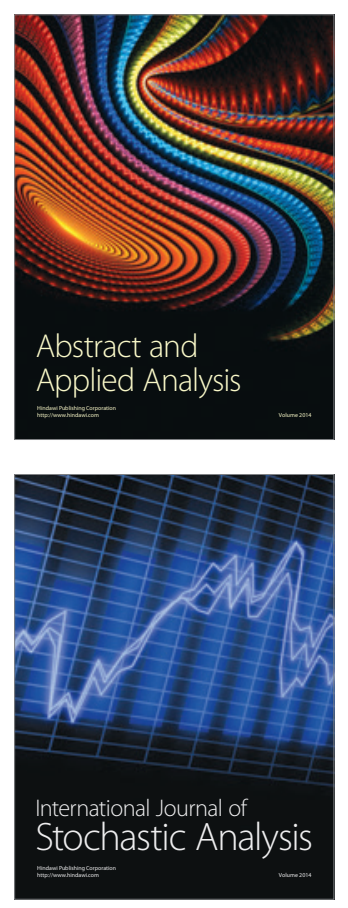

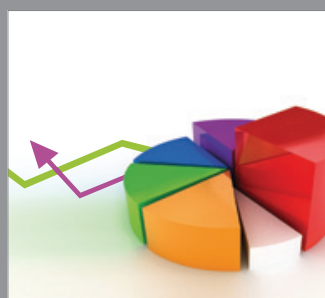

ournal of

Probability and Statistics

Promensencen
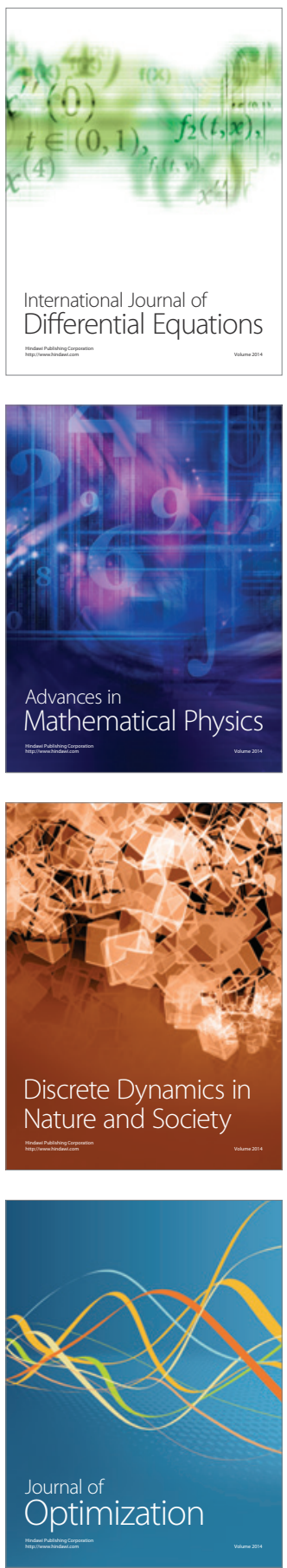